\title{
Properties of Blind Rendezvous in Channel Hopping Cognitive Piconets
}

\author{
Jelena Mišić, Vojislav B. Mišić, Mohammad Shahnoor Islam Khan, and Md. Mizanur Rahman \\ Ryerson University, Toronto, ON, Canada M5B 2K3.
}

\begin{abstract}
Rendezvous refers to the ability of cognitive nodes to find each other and form a network, or to find an already operating cognitive network and join it. It is a challenging problem, in particular in channel-hopping cognitive networks. In this paper, we discuss the performance of the probabilistic blind rendezvous mechanism based on the transmission tax-based MAC protocol with cooperative sensing at the MAC level. We investigate the performance of the algorithm, with particular focus on the difference in performance in an emergent vs. a fully operational channel hopping cognitive piconet.
\end{abstract}

\section{INTRODUCTION}

Channel hopping using a dynamically adaptive hopping sequence [13], similar to Bluetooth [11], [18], is a promising approach to avoid interference to and from licensed (primary) users in a cognitive network. Since this mode of operation resembles more traditional personal area networks, we refer to such networks as channel-hopping cognitive personal area networks, or CPANs in general, and to individual networks as piconets. One of the outstanding problems in CPAN development is the problem of rendezvous. Namely, to establish communication, a cognitive node must first attempt to meet another cognitive node (or an entire network) at the same RF channel and exchange synchronization data [10]. Channel hopping makes this problem particularly difficult because the individual hopping sequences of the nodes are not only different, but may change in time as well.

A number of approaches to the rendezvous problem have been proposed, with or without the aid of a dedicated infrastructure such as a central (base) station or a common control channel [7]. For obvious reasons, the latter approaches, collectively referred to as blind rendezvous [2], are preferable in practice. In this paper we investigate the performance of one such rendezvous mechanism [21], [22] developed in the context of the transmission-tax based MAC protocol [23] which incorporates spectrum sensing [19]. This mechanism ensures that rendezvous is achieved in the presence of primary user activity, and it does not require that the clocks of different devices are synchronized. Moreover, rendezvous can be achieved during piconet formation as well as during normal operation of the piconet; we refer to those two scenarios as the emergent and fully operational piconet case, respectively.

The rest of the paper is organized as follows: Section II surveys related work and highlights the rendezvous problem in more detail. Section III describes the probabilistic rendezvous mechanism in the transmission tax-based MAC protocol, while Section IV presents and compares rendezvous performance in an emergent and fully operational piconet case. Finally, Section $\mathrm{V}$ concludes the paper and highlights some future research.

\section{RELATED WORK}

In recent years a number of MAC protocols have been proposed for cognitive ad hoc and personal area networks [6]. Regarding the rendezvous problem, some solutions rely on the services of a central controller (e.g., [4]) or the availability of a dedicated common control channel [5], [17], [3], [7]. While both approaches promise good performance, i.e., short mean value and small (and provable) upper bound for time to rendezvous (TTR), their prerequisites - namely, a dedicated control channel or a central controller - are difficult to achieve in practice. Consequently, a blind rendezvous protocol is much better suited for truly distributed, autonomous cognitive networks [2].

Over time, two main approaches to blind rendezvous in cognitive networks have emerged. A large group of protocols rely on predefined deterministic channel hopping sequences [8], [9], [15], [16] (so it is questionable whether they should be considered to be truly blind). The necessary channel hopping sequences may be constructed in such a way that a finite upper bound for TTR is guaranteed, usually for two nodes finding each other, but sometimes even for multiple nodes finding others and establishing communication. However, most of these proposals suffer from the following shortcomings:

- First, they do not describe an actual rendezvous protocol, assuming instead that the rendezvous is accomplished when two nodes hop on a common available channel in the same time slot; obviously rendezvous can't be made if both nodes transmit or receive at the same time.

- Some approaches rely on the nodes' clocks and, by extension, their sequences, being synchronized - however, clock synchronization requires a central authority and, thus, it is not truly blind; furthermore, the clock phase shift (skew) may render the algorithm unusable.

- Most importantly, virtually all of sequence-based approaches simply ignore the presence of primary users and the impact of their activity - which is the basic tenet of cognitive communications. categories of blind rendezvous protocols have emerged over time.

- In addition, most papers focus on the scenario in which nodes just find each other and establish communication, instead of the scenario in which a node finds and joins an operational piconet. This approach is similar to that one 
adopted in Bluetooth [11], where the discovery procedure (as rendezvous is called in Bluetooth) is mutually exclusive with normal operation of the piconet. Such separation of rendezvous and normal operation is impractical in many scenarios such as emergency network operation, disaster management, military communications, and the like, where uninterrupted operation of the piconet is a must, yet new nodes should be allowed to join as soon as they appear in the vicinity.

We note that steps towards a piconet-oriented rendezvous protocol have been made, e.g., the network setup protocol described in [2], even this protocol depends on a central controller (called Cognitive Base Station) and does not entirely address the problem of avoiding interference from primary users.

On account of these shortcomings, rendezvous protocols in the other group appear to be more attractive in practice. Typically they rely on spectrum sensing and probabilistic channel selection, possibly aided by knowledge about primary user activity patterns obtained through some kind of learning [12], [10], [7], [22], [14]. Still, many of these protocols are not well integrated with the MAC protocol and require separation of rendezvous phase form normal operation. Recently, a MAC protocol has been described [20], [23] that may be easily extended to include a probabilistic rendezvous mechanism. This extension is the focus of the present paper.

\section{Probabilistic RENDEZVOUS IN TRANSMISSION TAX-BASED MAC PROTOCOL}

In the transmission tax-based MAC protocol, nodes are organized in piconets managed by a coordinator node, similar to Bluetooth [11]; as in Bluetooth, any node with sufficient computational capability may take up this role. Time is slotted into unit slots and organized in superframes, each of which takes place on a single channel; between two successive superframes, all nodes in the CPAN hop to the next channel as instructed by the coordinator. We assume that the superframe contains $s_{f}$ unit slots, some of which are reserved for administrative purposes such as reporting of sensing results, join/leave and bandwidth reservation requests, beacon and trailer frames. There is also a guard time during which all nodes hop to the next channel.

Each node can request time (i.e., bandwidth) for transmitting a number of up to $\mu$ data packets. Upon successful transmission, the sender node is obliged to perform sensing for $k_{p}$ superframes. Sensing nodes independently and randomly select which channels to sense during the data subframe of a superframe, and report the results back to the coordinator in the reporting subframe. The coordinator then compiles and updates a list of idle and busy channels - the channel mapand decides on the channel to be used for the next hop. Due to discrete character of sensing and the delay needed to collect the sensing results, the information in the coordinator's channel map differs from the actual state [19]; the sensing error may be controlled through judicious choice of $k_{p}$ [23].
Sensing duty may span several superframes when the transmission tax coefficient is greater than one. We assume that reporting is done in each of the $k_{p}$ superframes, which reduces the sensing error and improves throughout by allowing the sensing node to receive data when needed.

Many existing superframe-based MAC protocols require the beacon frame to be sent at the beginning of the superframe; however, the rendezvous protocol is better served by a trailing beacon or trailer. Namely, a node that overhears any valid frame in a superframe with a leading beacon will wait for the reservation sub-frame (which would then be the last one in the superframe) to send a join request. However, such a node could not know which channel to hop to in order to hear the next beacon, and thus would lose synchronization with the CPAN. A simple remedy would be to make the coordinator acknowledge a properly received join request packet with an ACK packet indicating the channel to be used for the next hop (and next superframe). However, should the join request packet or the coordinator's ACK packet get lost due to noise and/or interference, the node would still have no idea where to hop next and thus lose synchronization with the CPAN it has discovered only a moment ago.

The trailer includes bandwidth allocation for previously received transmission requests and the next-hop channel. It also includes announcements about join/leave requests granted by the coordinator, as explained in the next subsection.

We assume that all nodes are aware of the set of $N$ channels to be used. Initially, a cognitive node may spend some time trying to find if there is an operational CPAN in the vicinity; if such a CPAN is not found, the node will immediately begin to act as a coordinator, emitting beacon frames and hopping through available channels in a pseudo-random manner, trying to select idle channels (i.e., those without primary user activity) for its operation. Initially sensing will be performed by the coordinator; as other cognitive nodes join the CPAN using the rendezvous protocol, they will begin to exchange data and thus gradually take over the sensing function. However, basic functions of the CPAN (i.e., emitting beacon and trailer frames, bandwidth allocation, and admission of new nodes) will continue to be executed by the coordinator.

A newly arrived node that wants to find the CPAN (hereafter referred to simply as the node) must also hop randomly through the channels, as random hopping was shown to be the most efficient approach to rendezvous [1]. The node may hop to a channel which is busy, i.e., there is a primary user active on that channel; it will stay there for only a short time period, $T_{w b}$, which will be referred to as the busy timeout. The node may also hop to an idle channel, where it will stay for a longer time, hoping that the CPAN will eventually hop in to the same channel. The maximum residence interval in this case is referred to as the idle timeout, $T_{w i}$.

A new node that wants to find an operational CPAN may hop onto a busy channel, in which case it stay there for the duration of the busy timeout, $T_{w b}$. It may also hop to an idle channel, where it stays up to $T_{w i}$, the idle timeout, unless it makes the rendezvous or the channel becomes busy. 


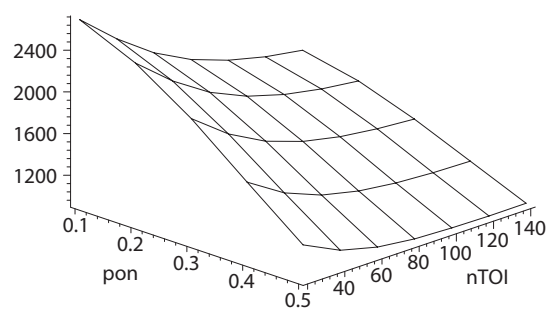

(a) Mean TTR when $N=25$ channels and mean primary cycle time is $\overline{T_{c y c}}=3000$.

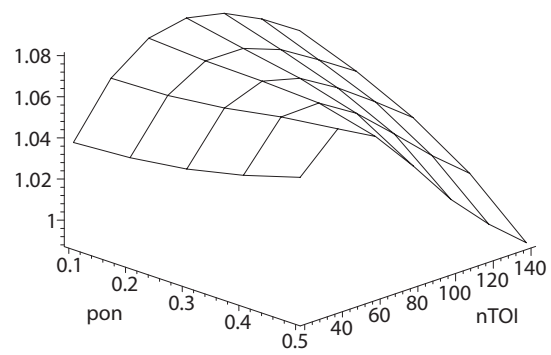

(d) Coefficient of variation of TTR when $N=$ 25 channels and mean primary cycle time is $\overline{T_{c y c}}=3000$.

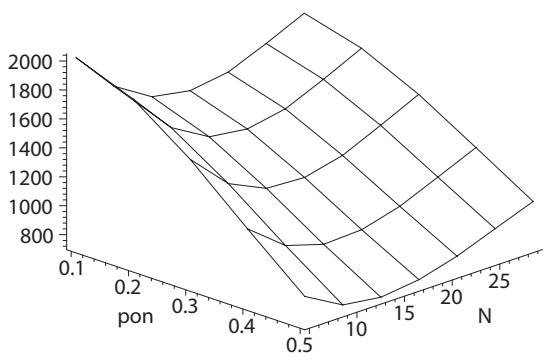

(b) Mean TTR when normalized idle timeout is $n T O I=140$ and mean primary cycle time is $\overline{T_{c y c}}=3000$.

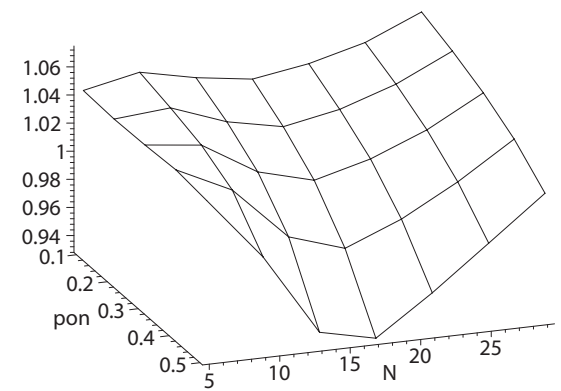

(e) Coefficient of variation of TTR when normalized idle timeout is $n T O I=140$ and mean primary cycle time is $\overline{T_{c y c}}=3000$.

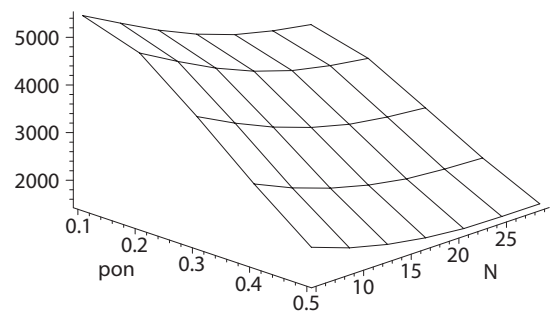

(c) Mean TTR when normalized idle timeout is $n T O I=140$ and mean primary cycle time is $\overline{T_{c y c}}=6000$.

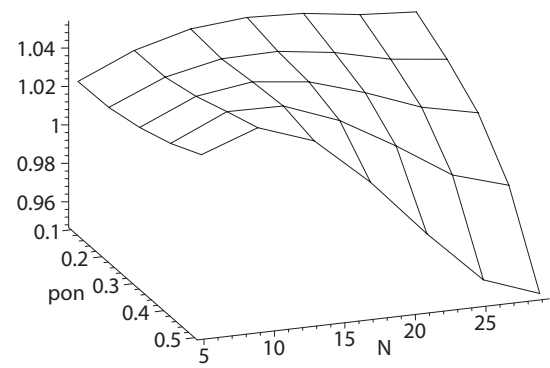

(f) Coefficient of variation of TTR when normalized idle timeout is $n T O I=140$ and mean primary cycle time is $\overline{T_{c y c}}=6000$.

Fig. 1. Rendezvous performance in the emergent piconet scenario: top row, mean TTR; bottom row, coefficient of variation of TTR.

Once the node hops in to the channel, it will begin to wait for CPAN transmissions. Rendezvous succeeds in the following scenarios.

In the simplest one, the CPAN hops in to the same channel and begins a superframe. The node recognizes the presence of the CPAN, waits for the reservation subframe, and sends a request to join the CPAN; the coordinator grants the request and announces the presence of the new node in the trailer. If the node has heard a transmission from a CPAN, it may prolong its stay beyond the time $T_{w i}$ in order to send its join request and hear the admission decision in the trailer. In the ideal case, all of these occurs without interference from a primary user transmission.

The node may also hop to the channel on which the CPAN superframe has already started. Rendezvous may be achieved as long as the node can send in the join request in the reservation subframe.

Finally, rendezvous can be achieved even if the node hops to the channel just in time to hear the trailer. The node will thus be able to follow the CPAN to the next hop and send its join request in the next superframe.

However, rendezvous can also fail, on account of the following. First, the CPAN superframe might be completed on the channel visited by the node before the arrival of the node to the channel.

Second, the node sojourn on an idle channel may exceed the its idle timeout so that the node leaves before the arrival of the CPAN.
Finally, the node and the CPAN may visit an idle channel and attempt to make a rendezvous, but the communication between the two and, consequently, the rendezvous are effectively destroyed by the onset of primary source activity. (Note that the onset of primary source activity will also destroy the ongoing CPAN superframe.)

Let us now analyze the performance of the rendezvous protocol.

\section{PERformance RESUlts FOR PROBABILISTIC RENDEZVOUS}

We have assumed that channel idle and busy times due to primary user activity are exponentially distributed with average values $\overline{T_{i}}$ and $\overline{T_{a}}$ respectively. Primary user activity factor $p_{o n}=\frac{\overline{T_{a}}}{\overline{T_{a}}+\overline{T_{i}}}$ was varied in the range 0.1 to 0.5 , which corresponds to low- to medium primary user activity. (A duty cycle of 0.5 means that mean durations of active and idle periods are equal.) The mean cycle time of primary user transmissions was set to $\overline{T_{c y c}}=\overline{T_{i}}+\overline{T_{a}}=3000$ or 6000 basic slots. For convenience and to avoid any over-reliance on an actual technology, all time intervals are normalized to a single sensing slot (basic slot).

Regarding the duration of the superframe, we have considered two cases. In the first case, hereafter referred to as emergent piconet, superframe duration was $s_{f}=50$ units, which fits the scenario in which the CPAN is in the process of build-up and there is no actual data exchange. In this scenario the piconet consists of just the coordinator which emits beacon 


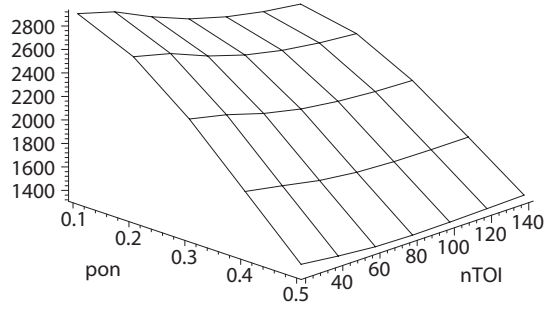

(a) Mean TTR when $N=25$ channels and mean primary cycle time is $\overline{T_{c y c}}=3000$.

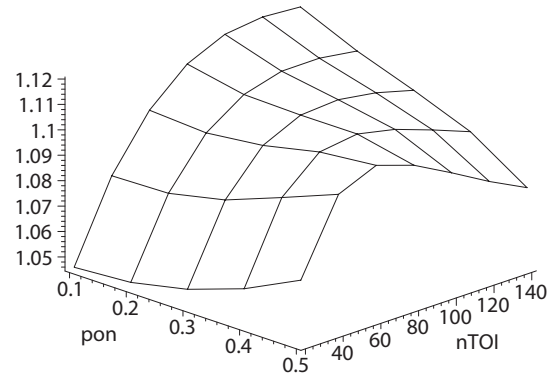

(d) Coefficient of variation of TTR when $N=$ 25 channels and mean primary cycle time is $\overline{T_{c y c}}=3000$.

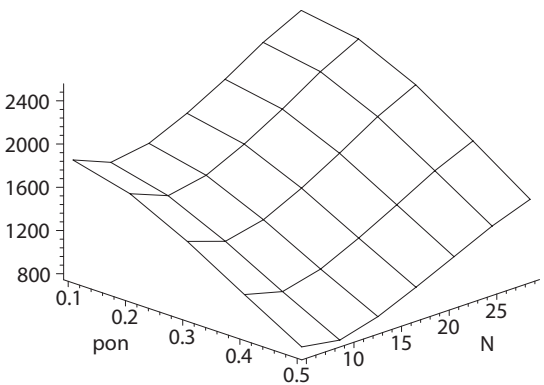

(b) Mean TTR when normalized idle timeout is $n T O I=140$ and mean primary cycle time is $\overline{T_{c y c}}=3000$.

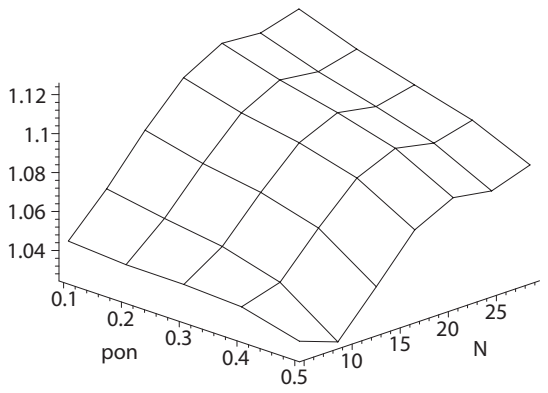

(e) Coefficient of variation of TTR when normalized idle timeout is $n T O I=140$ and mean primary cycle time is $\overline{T_{c y c}}=3000$.

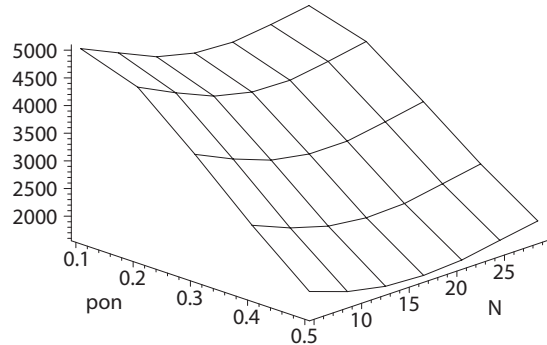

(c) Mean TTR when normalized idle timeout is $n T O I=140$ and mean primary cycle time is $\overline{T_{c y c}}=6000$.

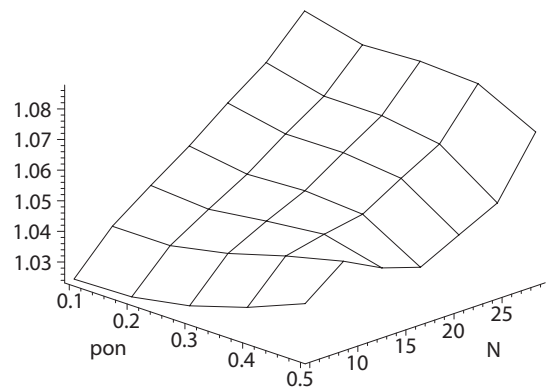

(f) Coefficient of variation of TTR when normalized idle timeout is $n T O I=140$ and mean primary cycle time is $\overline{T_{c y c}}=6000$.

Fig. 2. Rendezvous performance in the operational piconet scenario: top row, mean TTR; bottom row, coefficient of variation of TTR.

and trailer frames. Since there is no data traffic, the superframe duration was set to $s_{f}=50$ basic slots. A portion of that time, lasting for $\Delta=20$ units, was set aside for reservation and join requests, beacon, trailer, and guard intervals. The coordinator performs the sensing itself during the data transmission subframe; sensing of one channel, including the time needed to switch to the channel, was assumed to take $d_{s}=5$ slots.

We have also set up an experiment where a node attempts to find and join a CPAN piconet which fully operational. In this case, the piconet has the superframe duration of $s_{f}=$ 100 slots and $M=16$ nodes, each having a buffer of size $K=10$ packets. Packet arrival process was set to Poisson with arrival rate of $\lambda=0.002$ packets per slot per node, while packet duration was uniformly distributed between 8 and 12 time units with an average value of $k_{d}=10$. Duration of the acknowledgment packet was set to one time unit. Packet destinations were uniformly distributed over all piconet nodes. Maximum number of packets from a single node that can be serviced in one superframe is $\mu=3$. Transmission tax was set to $k_{p}=4$ superframes per transmission, regardless of the number of packets sent.

In both cases, the parameters of the rendezvous protocol were set as follows: the busy timeout was fixed at $T_{w b}=10$ unit slots, while the idle timeout was set to the product of the number of primary channels and normalized timeout parameter $n T O I$, i.e., $T_{w i}=n T O I \cdot N$.

The diagrams in Fig. 1 shows main performance descriptors of the probabilistic algorithm in the emergent piconet scenario. The diagrams in the top row present the mean TTR, while those in the bottom row show the coefficient of variation of TTR. As can be seen from the diagrams in the leftmost column, mean TTR decreases with an increase in primary user duty cycle. This is not unexpected since larger value of duty cycle correspond to shorter idle time which, in turn, increases the probability of overlap between CPAN and node residence times conditioned on their meeting at an idle channel. At the same time, mean TTR decreases when normalized timeout $n T O I$ increases; the effect is more pronounced at larger channel idle times. Coefficient of variation of rendezvous time is in the range of 1 to 1.08 which indicates that the distribution of rendezvous time is mildly hyperexponential.

On account of these results, we have chosen to use the value of $n T O I=140$ for the normalized idle timeout of the probabilistic rendezvous algorithm in further experiments.

The other two sets of diagrams show the impact of primary user cycle time under variable number of channels and primary user duty cycle. At smaller cycle time $\overline{T_{c y c}}=3000$, TTR has a minimum around 13 channels, while for $\overline{T_{c y c}}=6000$ rendezvous time monotonically decreases when the number of channels increases. Minimal mean TTR is also accompanied with sub-exponential values of the coefficient of variation of TTR.

Basic performance descriptors for the operational piconet scenario are shown in Fig. 2; as above, the diagrams in the top 
row present the mean Time-to-rendezvous (TTR), while those in the bottom row show the coefficient of variation of TTR. As can be seen, mean TTR and its coefficient of variation increase only slightly in comparison with the emergent piconet, mainly on account of longer superframe. Also, the coefficient of variation takes values in the range 1.02 to 1.12 , which makes the distribution of TTR mildly hyperexponential.

When the number of channels increases, more channels become available for both the node and the CPAN to choose from; as the result, the mean TTR increases. Coefficient of variation of rendezvous time slightly increases with the number of channels and decreases when activity factor is growing, which is expected.

When the cycle time of primary source grows, mean TTR will also increase; however, coefficient of variation is slightly smaller when the channel cycle time increases since variation of unsuccessful waiting time is becoming smaller.

On the whole, performance of the probabilistic rendezvous is only slightly worse in the fully operational piconet scenario vs. that in the emergent piconet scenario. However, the operational piconet scenario allows the channel-hopping cognitive piconet to operate normally while still accepting new nodes, which would be an extremely desirable capability in many applications such as emergency/disaster response and others.

While the probabilistic rendezvous is a truly blind mechanism that can't ensure a guaranteed mean TTR - unlike its sequence-based counterparts discussed in Section II - it is remarkably resilient to random primary user activity patterns. Moreover, the fact that its coefficient of variation is reasonably close to 1 , thus indicating that its probability distribution is close to exponential, means that in practice such protocol would provide predictable performance in a wide range of primary user traffic.

\section{CONCLUSiON}

In this paper we have proposed a probabilistic rendezvous algorithm in the context of a simple transmission tax-based MAC protocol for channel hopping cognitive personal area networks, and evaluated its performance in the context of an emergent piconet (which corresponds to the case when two cognitive nodes look for each other in order to establish communication) and an operational piconet which a newly arrived cognitive node wants to find and join. Mean TTR is shown to depend mostly on the number of channels and primary user activity factor, and to a somewhat lesser extent to the primary user cycle time.

Our future work will focus on practical implementations and tuning of these algorithms, including suitable recovery algorithms that will allow a transmission-tax based CPAN piconet to resume normal operation upon collision with primary user transmission. We also plan to work on estimation of primary user activity patterns, esp. in cases where primary user activity does not follow a memoryless probability distribution.

\section{REFERENCES}

[1] E. Anderson and R. Weber. The rendezvous problem on discrete locations. J. Applied Probability and Statistics, 28:839-851, 1990.
[2] C. J. L. Arachchige, S. Venkatesan, and N. Mittal. An asynchronous neighbor discovery algorithm for cognitive radio networks. In 3rd IEEE Int'l Symp. on New Frontiers in Dynamic Spectrum Access Networks DySPAN 2008, Chicago, IL, Oct. 2008.

[3] K. Bian, J.-M. Park, and R. Chen. Control channel establishment in cognitive radio networks using channel hopping. IEEE J. on Selected Areas in Communications - Wireless Series, 29(4):689-703, 2011.

[4] V. Brik, E. Rozner, S. Banerjee, and P. Bahl. DSAP: a protocol for coordinated spectrum access. In IEEE Int'l Symp. on New Frontiers in Dynamic Spectrum Access Networks DySPAN 2005, pages 611-614, Baltimore, MD, Nov. 2005.

[5] C. Cordeiro, K. Challapali, D. Birru, and N. Sai Shankar. IEEE 802.22: the first worldwide wireless standard based on cognitive radios. In IEEE Int'l Symp. on New Frontiers in Dynamic Spectrum Access Networks DySPAN 2005, pages 328-337, Baltimore, MD, Nov. 2005.

[6] C. Cormio and K. R. Chowdhury. A survey on MAC protocols for cognitive radio networks. Ad Hoc Networks, 7(7):1315-1329, 2009.

[7] C. Cormio and K. R. Chowdhury. Common control channel design for cognitive radio wireless ad hoc networks using adaptive frequency hopping. Ad Hoc Networks, 8(4):430-438, 2010.

[8] L. DaSilva and I. Guerreiro. Sequence-based rendezvous for dynamic spectrum access. In 3rd IEEE Symposium on New Frontiers in Dynamic Spectrum Access Networks (DySPAN 2008), pages 1-7, Oct. 2008.

[9] Y. Duan, G. Liu, and Z. Cai. Opportunistic channel-hopping based effective rendezvous establishment in cognitive radio networks. In Wireless Algorithms, Systems, and Applications, pages 324-336, 2012.

[10] B. Horine and D. Turgut. Link rendezvous protocol for cognitive radio networks. In 2nd IEEE Int'l Symp. on New Frontiers in Dynamic Spectrum Access Networks DySPAN 2007, pages 444-447, Dublin, Ireland, Apr. 2007.

[11] IEEE. Wireless medium access control (MAC) and physical layer (PHY) specifications for wireless personal area networks (WPAN). IEEE Std 802.15.1 (Revision of IEEE Std 802.15.1-2002), IEEE, New York, NY, 2005.

[12] Y. Kondareddy, P. Agrawal, and K. Sivalingam. Cognitive radio network setup without a common control channel. In IEEE Military Communications Conference (MILCOM 2008), pages 1-6, 2008.

[13] P. K. Lee. Joint frequency hopping and adaptive spectrum exploitation. In IEEE Military Communications Conference MILCOM2001, volume 1, pages 566-570, Washington, DC, Oct. 2001.

[14] B. Li, P. Yang, J. Wang, L. Shen, Y. Xu, Y. Liu, and X.-Y. Li. Optimal action point for dynamic spectrum utilization under Rayleigh fading. Ad Hoc and Sensor Wireless Networks, 17(1-2):1-32, 2013.

[15] Z. Lin, H. Liu, X. Chu, and Y.-W. Leung. Ring-walk rendezvous algorithms for cognitive radio networks. Ad Hoc and Sensor Wireless Networks, 16(4):243-271, 2012.

[16] H. Liu, Z. Lin, X. Chu, and Y.-W. Leung. Jump-stay rendezvous algorithm for cognitive radio networks. IEEE Transactions on Parallel and Distributed Systems, 23(10):1867-1881, Oct. 2012.

[17] L. Ma, X. Han, and C.-C. Shen. Dynamic open spectrum sharing MAC protocol for wireless ad hoc networks. In IEEE Int'l Symp. on New Frontiers in Dynamic Spectrum Access Networks DySPAN 2005, pages 203-213, Baltimore, MD, Nov. 2005.

[18] J. Mišić and V. B. Mišić. Performance Modeling and Analysis of Bluetooth Networks: Network Formation, Polling, Scheduling, and Traffic Control. CRC Press, Boca Raton, FL, July 2005.

[19] J. Mišić and V. B. Mišić. Performance of cooperative sensing at the MAC level: Error minimization through differential sensing. IEEE Transactions on Vehicular Technology, 58(5):2457-2470, June 2009.

[20] J. Mišić and V. B. Mišić. Simple and efficient MAC for cognitive wireless personal area networks. In Proc. Global Telecommunications Conference GLOBECOM'09, Honolulu, HI, Nov. 2009.

[21] J. Mišić, N. Khan, H. Khojasteh, and V. B. Mišić. CSCD: a simple channel scan protocol to discover and join a cognitive PAN. In IEEE Wireless Communications and Networking Conference (WCNC'2012), Paris, France, Apr. 2012.

[22] J. Mišić, H. Khojasteh, N. Khan, and V. B. Mišić. Towards an efficient rendezvous protocol for a cognitive PAN. In IEEE International Conference on Communications (ICC'2012), Ottawa, ON, June 2012.

[23] V. B. Mišić and J. Mišić. Cognitive MAC protocol with transmission tax: Dynamically adjusting sensing and data performance. In Proc. Global Telecommunications Conference GLOBECOM'10, Miami, FL, Dec. 2010. 